\title{
Personalized Trusted Service Recommendation Method based on Social Work
}

\author{
Du Ruizhong, Pei Zhi and Tian Junfeng \\ School of Computer Science and Technology, University of Hebei, \\ BaoDing 071002, China \\ drzh@mail.hbu.edu.cn
}

\begin{abstract}
In order to improve credibility and precision of the personalized service recommendation, we propose a personalized trusted services recommendation method based on social network. Firstly, according to the similarity of user's preference, the method build new relations combined with direct trust and indirect trust of users between them. Secondly, form a set of similar services which includes services invoked by target users. Eventually, get user's indirect trustrank to the recommended services through the weighted average of the target users' indirect trustrank to the recommended service and thus form a recommendation list. The experiment take precision rate, recall rate and F1_Measure as evaluation indexes. Comparing with traditional method which based on no confidence and users' trusted network, this approach demonstrates its effectiveness and feasibility through the result of the experiment.
\end{abstract}

Keywords: preference similarity, direct trust, a set of similar services, indirect trust

\section{Introduction}

With rapid development of Web service on the Internet, social network as a socializing tool has been widely used in recent years, such as Facebook, Renren and Friend circle. People check them everyday to make friends and update thoughts. Meanwhile, social network can be more useful to the recommendation system, because people tend to trust their friends' recommendation. The service experience can be shared among friends on social network. A increasing number of companies are promoting themselves through it because it's fast, concentrated and direct. In order to attract more consumers, companies often introduce some promotions that people just need to forward or like to get a free gift or discount. However, people usually help advertising the company without knowing it for getting profits. Although the credibility of friends' recommendation on social network is increasing, it is still not high enough if the friends don't know about the service they recommended. Therefore, using social networks between friends can be resolved for the new user's "cold start" problem, and we proposed user's trust to services to improve the credibility of the recommended services.

\section{Background and Related Work}

Driven by social networks, users' describe not only includes the service functions in aspects of functional and non-functional, but also ratings and feedbacks. How to dig out the trust relationship between users and users' personalized preferences from friends list and history behaviors is particularly important for using collaborative filtering approach to recommend for users ${ }^{[1]}$. J. Bobadilla et al. ${ }^{[2]}$ conducted a survey on collaborative filtering recommendation system method, provided an overview of the recommended system, and explained their original classification and evolution. Gan Zaobin et al. ${ }^{[3]}$ multi-dimensional reputation based on trust computation algorithm proposed trust evaluation for e-commerce transactions from former behaviors. N. Sánchez-Pi et al. ${ }^{[4]}$, 
who proposed a context-aware knowledge-based approach, used methods of CommonKADS represented context information and designed, defined and evaluated the system. Chen Xi et al. ${ }^{[5]}$ proposed a innovative collaborative filtering recommendation algorithm based QoS of Web services, provided a personalized map to scan the recommend results. That showed clearly the relationship between the QoS of Web services which recommended. Zhu Qiang et al. ${ }^{[6]}$ proposed a trust-based collaborative filtering method to calculate the degree of trust between users by constructing the social network to analyze the users' relations and interactive behaviors. And then to divide the community of friends and application services to recommended. Wang Haiyan, et al. ${ }^{[7]}$ proposed a method based on trust coalition, which taked into account that different property characteristics of services may produce different effects in the similarity metric, and proposed the trust of the target user's recommended behavior. Zhang Peiyun et al. ${ }^{[8]}$ proposed a recommended method of social network based on trust relationship. It used direct trust between the users and society network nodes' direct trust to the services to calculate the target user to the recommended services. Furthermost, the method calculated the relevance of them to improve the recommendation performance. Deng Shuiguang et al. ${ }^{[9]}$ proposed a network point of view on the personalized service recommended, which took into account not only the similarity of the user's preferences, but also considering the similarity of the services. More research works from different angles to make service recommended, that make the personalized trusted service recommended lay a solid foundation. But their consideration is not comprehensive enough in some cases. Either for users' trust, or does not consider users' preferences. And others without considering the similarity of the service or users' trust to the services.

In real life, some malicious users raise some interest deliberately or denigrate a service in order to mimic the target user's interests to achieve a higher similarity of preference. The recommendation system will recommend a malicious user's services to the target user. That makes them become candidate recommended neighbors, and lead to reduce the precision of the recommended results. Thus, friends with similar interests can effectively prevent malicious users recommend. We propose a personalized trusted service recommended method based on social network, it can be recommended by friends or his friend's friends. At the same time, in order to avoid making distrust publicity recommended that the friends without using the service, we use the direct trust of the users to the services, and calculate a set of similar services which the target users had invoked.

\section{The Trust Relationship}

\subsection{Direct Trust between Users}

In social network, a newly registered user will firstly expand his circle of friends by adding friends with same location or experiences. This trust is generated by similarity. After adding friends, clients and friends will have long and frequent direct interaction behaviors such as chatting, to increase trust. This trust is generated by the familiarity. Thus, the direct trust between users is generated by both similarity and familiarity, which can be expressed as:

$$
T_{d}(j, i)=n T_{\text {sim }}(j, i)+(1-n) T_{\text {chat }}(j, i)
$$

$T_{d}(j, i)$ represents the direct trust from user $j$ to his friend $i$, which ranges in $[0,1]$. $\mathrm{T}_{\text {sim }}(\mathrm{j}, \mathrm{i})$ represents the trust from user $\mathrm{j}$ to his friend $\mathrm{i}$ caused by similarity, which ranges in $[0,1] . T_{\text {chat }}(j, i)$ represents the trust from user $j$ to his friend $i$ caused by familiarity, which ranges in $[0,1] . \mathrm{n}$ is the adjustment factor. 
Usually, friends of same age share same interests, friends in same location have enjoy same service quality. So, we can calculate the similarity of trust through its attributes before they have no direct interaction.

Among direct interactions between friends, sending messages is the most common way to gain familiarity. However, sending messages is a mutual act, if one keep sending messages without the other's response, that may be harassment. So, when calculating the trust caused by chatting, it is more reasonable to take the number of messages sent out by the users to his friends. Because the relationship between sent messages and familiar trust conform the theory of diminishing marginal effect, so $\log$ is used to measure the relationship between them. That can be calculated as:

$T_{\text {chat }}(j, i)=\ln \left(\frac{\text { num }_{j i}}{\sum_{k \in U_{j}} n u m_{j k}}+1\right), \quad d(j, i)=1$

$\mathrm{T}_{\text {chat }}$ represents the trust from user $\mathrm{j}$ to his friend $\mathrm{i}$ by familiar. num $\mathrm{j}_{\mathrm{ji}}$ represents the number of messages sent by user $j$ to his friend i. $U_{j}$ represents the set of user $j$ 's friends with whom user $\mathrm{j}$ chat. num $\mathrm{j}_{\mathrm{jk}}$ represents the total number of the messages sent by user $\mathrm{j}$ in chat with his friends. $d(j, i)=1$ represents user $j$ and $i$ are friend when their distance is 1 .

\subsection{Indirect Trust between Users}

In social networks, users often have interactive relationship with their friends for a long time, and trust can be spread in the social network. For example, if user A trusted user B and user B trusted user $\mathrm{C}$, so user $\mathrm{C}$ has a certain trust to user $\mathrm{A}$. Therefore, there are at least one trust path would connect two strangers in social network, and the distance between them is greater than 1 . We called it indirect trust.

When there are multiple trust paths between two users, we select the highest direct trust to target users, as shown in Figure 1. When the user j's direct trust to the user A is greater than $\mathrm{B}$, select $\mathrm{A}$. When the target user to the nearest users has the same direct trust, the shortest trust path will be chosen. For example, the user j's direct trust to A is equal to C, but the trust path to user $\mathrm{c}$ is the shortest. When there are multiple shortest paths, take the greater value in the final calculation as indirect trust between users. Assume that $p(j, c, i)$ from user $\mathrm{j}$ to user $\mathrm{i}$ is the shortest path, then the indirect trust is calculated as $_{\mathrm{jc}} * \mathrm{~T}_{\mathrm{c}}$, as shown in Figure 1.

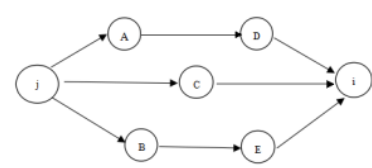

Figure 1. Indirect Trust Relationship between the User i and the User j

\subsection{Preference Trust between Users}

Although recommending service base on users' trust can increase its credibility, trusted users' different preferences lead to the result credible but not always likable by people. Therefore, we came up with a kind of preference trust relationship between the users that combined trust relationship with preference similarity.

Suppose user j's trust to user $i$ is $T_{u}(j, i)$ and there preference similarity is $\operatorname{Sim} U(i, j)$. So, the preference trust from user $\mathrm{j}$ to user $\mathrm{i}$ is $\mathrm{T}_{\text {pref }}(\mathrm{j}, \mathrm{i})^{[10]}$. With higher trust and preference similarity, the preference trust between the users becomes higher. If the trust is zero or they are perfectly negatively correlated, the preference trust between users should be zero. That can be expressed as follows:

$T_{\text {pref }}(j, i)=\frac{2 T_{u}(j, i) \cdot \operatorname{Sim} U(i, j)}{T_{u}(j, i)+\operatorname{Sim} U(i, j)}$ 


\subsection{Users' Direct Trust to Service}

If the service has not been invoked by users, then it is unable to determine the trust degree, and an initial value will be appointed to it. After the target user invoked the service, there will be a feedback about quality of the service, and increase or decrease to the trust degree is depends on it. Trust updated to the next goal as a recommended user's direct trust. We name the trust degree as directly trust degree which bring out by invoking service directly.

Service $\mathrm{s}$ is other users recommend to the target user, if the user $\mathrm{i}$ have not been invoked the service $\mathrm{s}$, the trust for it is other users' indirect trust expressed as $\mathrm{V}_{\mathrm{n}}(\mathrm{i}, \mathrm{s})$. If the user had invoked the service, it would have feedback rating expressed as $R_{i s}$, which range in $[1, n]$, where $\mathrm{n}$ is the maximum level. At the same time, that cause direct trust $\mathrm{V}_{\mathrm{n}+1}(\mathrm{i}, \mathrm{s})$ which range in $[0,1]$. If user i successful invoked the service $\mathrm{s}$ and the trust value is 1 , so the direct trust updated is averaging the sum expressed as: $V_{n+1}(i, s)=\left(V_{n}(i, s)+1\right) / 2$.

If user $\mathrm{i}$ invoked the service $\mathrm{s}$ is failed, then its trust value half which can expressed as: $V_{n+1}(i, s)=V_{n}(i, s) / 2$.

This method can quickly reduce trust value of the invoked failed services, and the losing value require a successful invoked to regain in many times.

\section{The Similarity Calculation}

\subsection{Similarity Calculation between Services}

The similarity between the services can be calculated by the same user to different services' feedback ratings. In the process of recommendation services, the services invoked by the target user can form a set of similar services through the service similarity. Recommend services from the set of similar services, it can improve the problem of data sparseness.

Collect history of the user invoking the service to generate scoring matrix $\mathrm{R}_{\mathrm{m}^{*} \mathrm{n}}$ :

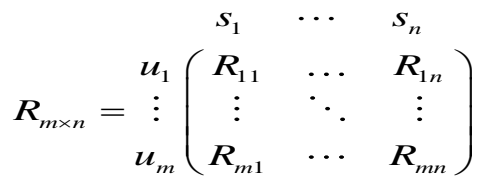

Among them, each row represents a user, each column represents a service. According to Pearson correlation coefficient, service $s_{i}$ and $s_{j}$ are rated by the same user, the similarity is calculated as follows:

$$
\operatorname{SimS}\left(s_{i}, s_{j}\right)=\frac{\sum_{i \in U_{c}}\left(R_{i_{i}}-\overline{R_{s_{i}}}\right)\left(R_{i s_{j}}-\overline{R_{s_{j}}}\right)}{\sqrt{\sum_{i \in U_{c}}\left(R_{i s_{i}}-\overline{R_{s_{i}}}\right)^{2}} \sqrt{\sum_{i \in U_{c}}\left(R_{i s_{j}}-\bar{R}_{s_{j}}\right)^{2}}}
$$

Pearson correlation coefficient indicates score relevant between the two services. The larger the value, the higher the degree of similarity service $s_{i}$ and $s_{j} . U_{c}$ is a set of users who rate service $s_{i}$ and service $s_{j}$ commonly. $R_{i s i}$ and $R_{i s j}$ are the users of the service $s_{i}$ and $\mathrm{s}_{\mathrm{j}}$ ratings, respectively. $\overline{R s i}$ Rsi and $\overline{R s j}$ are the average score that service $\mathrm{s}_{\mathrm{i}}$ and $\mathrm{s}_{\mathrm{j}}$ are invoked by the same set of users, as shown in Figure 2. 


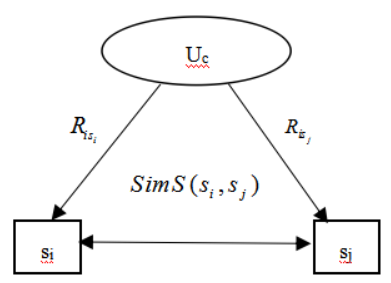

Figure 2. Service $s_{i}$ and $s_{j}$ are Commonly Rated by a Set of Users $U_{c}$

\subsection{Preference Similarity Calculation between Users}

Trust between users can improve the user's credibility, but high reliability between friends maybe has different preferences. For example, user A like to play games at home, and his friend B loves to travel. Although the user A and his friend B are trust, the user A recommend to the user $\mathrm{B}$ a game, the user $\mathrm{B}$ may not like it. While the user A recommend to his friend $\mathrm{C}$ who loves to play games maybe like it. Therefore, when considering the trust between the users as well as considering the similarity preferences between them.

Assume that the user $\mathrm{i}$ and the user $\mathrm{j}$ are both invoked the service $\mathrm{s}$, according to Pearson correlation coefficient, the preference similarity of them as follows:

$\operatorname{Sim} U(i, j)=\frac{\sum_{s \in S_{u}}\left(R_{i s}-\overline{R_{i}}\right)\left(R_{j s}-\overline{R_{j}}\right)}{\sqrt{\sum_{s \in S_{u}}\left(R_{i s}-\overline{R_{i}}\right)^{2}} \sqrt{\sum_{s \in S_{u}}\left(R_{j s}-\overline{R_{j}}\right)^{2}}}$

$S_{u}$ is the user $i$ and the user $j$ both invoked the historical set of services. $R_{i s}$ and $R_{j s}$ are history rated by them. $\bar{R}_{i}$ and ${ }^{\bar{R}_{j}}$ are the average value by the user $\mathrm{i}$ and $\mathrm{j}$ commonly rated for the set of services $S_{u}$.

\section{Indirect Trust of the Target User to the Recommendation Services}

Definition: indirect trust of the target user to the recommendation services. It refers to the target user's trust to the recommendation services is generated by other trust users direct invoked the services.

Assume that $U_{c}$ is the set of recommendation users. A user i from $U_{c}$ directly invoked a service from the set of similar services $S_{k}$, and the direct trust is $V(i, s)$. Recommend the service to the target user, and there preference trust as weights which is $T_{\text {pref }}(j, i)$. Target users $\mathrm{j}$ 's indirect trust to the service $s$ is a weighted average of the direct trust which is $\mathrm{V}_{\mathrm{u}}(\mathrm{j}, \mathrm{s})$ calculated as follows:

$V_{u}(j, s)=\frac{\sum_{i \in U_{c}} T_{\text {pref }}(j, i) V(i, s)}{\sum_{i \in U_{c}} T_{\text {pref }}(j, i)}$

\section{Personalized Recommendation Algorithm Trusted Service}

(1) Choose anyone from candidate neighbors for the target user $\mathrm{j}$, and calculate preference similarity of the target user $\mathrm{j}$ with recommendation user $\mathrm{i}$ by using equation (5).

(2) Calculate the trust from user $\mathrm{j}$ to recommendation user $\mathrm{i}$.

(3) Calculate the preference trust from target user $\mathrm{j}$ to recommend user $\mathrm{i}$ by using equation (3).

(4) Make the preference trust in descending order, and select the top-M recommendation users to form a set of recommendation users as $\mathrm{U}_{\mathrm{c}}=\left\{\mathrm{u}_{1}, \mathrm{u}_{2}, \ldots, \mathrm{u}_{\mathrm{m}}\right\}$. 
(5) For the service $s_{j}$ which invoked by target user $j$, we calculate some similar services with the service $s_{j}$ which invoked by users from $U_{c}$, and form a set of similar services called $S_{k}$ by using equation (4).

(6) According to preference trust and direct trust, we calculate the indirect trust of target user $\mathrm{j}$ to user $\mathrm{i}$ by using equation (6).

(7) Make the services from $S_{k}$ arranged in descending by indirect trust, and the top-N services from $S_{\mathrm{k}}$ return to be recommended.

\section{Experiment and Analysis}

To verify the service recommendation method, the experiment use the University of Minnesota recommendation system research team's movie ratings data sets called MovieLens. This data sets contains 943 users and 1682 movie ratings data, evaluation of every user at least 20 films and ratings are 1-5. The ratings reflect preferences of users of its degree that 1 is divided into a minimum and 5 being the highest. The MovieLens data randomly set $20 \%$ data as a test set, and the remaining 80 percent of the data as a training set. Build trust relationships between users and each user has a buddy list. The user attributes as age, location and the number of messages which chatting with friends. The similarity and familiarity are the same weight on the experiment. The number of candidates recommendation take 50 users and the number of similar services take 20 services. The initial trust of the recommendation users to the service is 0.6 , and referral service number as 5 to 15 in steps of 1 .

Experiments using Java programming language, the experimental environment for the Intel Core 2 Duo processor, CPU clocked at $2.93 \mathrm{GHz}, 3 \mathrm{~GB}$ memory of the PC, Windows 7 operating system, and development platform Eclipse 4.4.

\subsection{The Users' Trust}

When calculate the users' trust resulting from the similarity, people think that the same age group have similar preference. At the same time, the smaller the user's administrative region are, the higher similar to the experience of quality of service at the same familiarity of them. Therefore, the age and the location represented users' attributes at the same weight. The user $\mathrm{j}$ 's trust to the user $\mathrm{i}$ generated by similarity can be expressed as: $T_{\text {sim }}(j, i)=\left(T_{\text {age }}(j, i)+T_{\text {location }}(j, i)\right) / 2$.

The experiment take a difference of 10 years old as an age group, otherwise, the similar trust value is 0 . It can be expressed as: $T_{a g e}=1-\left|A g e_{i}-A_{g e}\right||10| A, g e_{i}-A_{g e} \mid \leq 10$. When the location between users are from the same countries, Tlocation $(j, i)$ is 0.1 . When the same province is 0.3 , the same city is 0.5 , the same county is 0.8 , the same street is 0.9 , the same cell is 1 , and the others is 0 .

\subsection{Precision}

Precision is a measure of the precise of the recommendation method to predict the degree of favorite for a target user to recommendation services, and to show the proportion of the service actually like for users recommended to the services by recommendation system. There are four kinds of recommendation results if a user has not invoked a service, which namely system recommend and users like called True Positive (TP), system recommend but users do not like called False Positive (FP), system does not recommend but users like called False Negative (FN), and system is not recommend and users do not like called True Negative (TN), as shown in Table 1:

For user i, its recommendation precision rate is the top-N services which the users prefer proportion of services by recommendation system, namely: 


$$
P_{i}(N)=\frac{T P}{T P+F P}
$$

\subsection{Recall}

Recall is a classification accuracy of assessment methods, which represents the probability of services recommended which the user like, defined as the proportion of the services the user prefer on the recommendation list that in all service users prefer, namely:

$$
R_{i}(N)=\frac{T P}{T P+F N}
$$

\subsection{F1_Measure Index}

Using precision and recall rate alone are not a good assessment of the recommendation system, because they are dependent on the recommendation list, and a negative correlation between them. With an increase of the recommendation length of the list, the precision would decrease while the recall rate increases. So, when a system is not a fixed list of recommendation length, we need a containing precision and recall rate twodimensional vector called F1_Measure index ${ }^{[11]}$ to reflect the performance of the system:

$$
F 1 \_\operatorname{Measure}(N)=\frac{2 P(N) R(N)}{P(N)+R(N)}
$$

\subsection{Analysis of Experimental Results}

Using precision, recall and F1_Measure index were comparative analyzed personalized trusted service recommendation method based on social networking (PTSR), recommendation method based on users' trust networks ${ }^{[12]}$ (UTR), and service recommendation method based on the non-trust ${ }^{[13]}$ (NTR), in Figure 3-5.

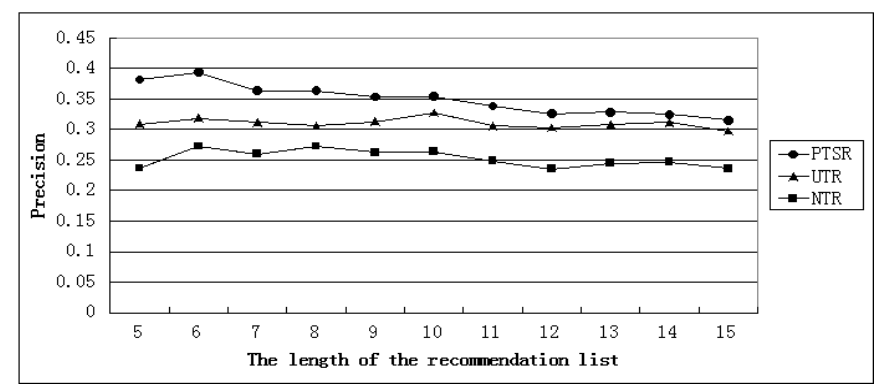

Figure 3. Influence Recommendation List of Top-N for Recall

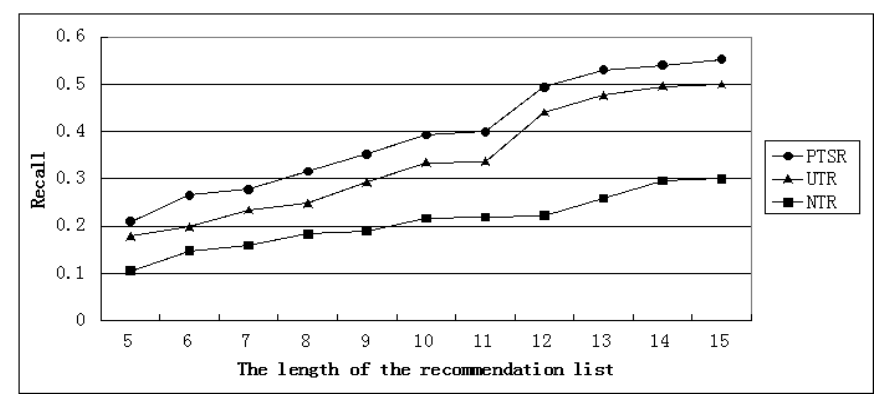

Figure 4. Influence Recommendation List of Top-N for Recall 


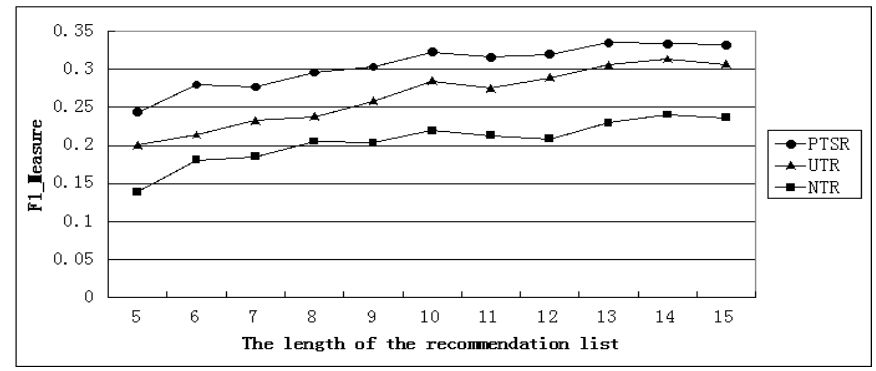

Figure 5. F1_Measure Index

From the experimental results, with the increase of the length for recommendation list, the precision of the three methods all decrease while the recall rate has a certain upgrade. It shows that both of them are negative correlation with increasing the length of the recommendation list. From the F1_Measure index we can see the recommendation method based on trust is better than non-trust recommendation. Personalized trusted service recommendation method results superior recommendation method based on the user trust networks. This is because the latter only consider the trust network in the target user as a trusted user's most recent rate of reference, and lost the trust path from the larger trust path, while ignoring the user to the services' trust and services' similarity. In summary, through the analysis of three methods of indicators, not only it shows the importance of trust between users, user to service, but also verify the effectiveness and feasibility of the proposed method.

\section{Conclusion}

To improve credibility and precision of the personalized service recommendation, combine the trust among users and preference similarity between users to form preferences trust between users. That increases credibility of the service recommendation by users' direct trust to the service, and improves precision of the service recommendation through the set of similarity services. Finally, the experiment shows effectiveness of the algorithm. It is necessary to follow-up work in studying deeply on the dynamic social networking environment and the recommendation mechanism in the cloud computing environment to further improve service recommended performance.

\section{Acknowledgements}

This work was supported in part by The National Natural Science Foundation of China $(61170254,60873203)$, the natural science foundation of Hebei province (F2014201098, F2014201099), the university of Hebei province science and technology research program (2013-250).

\section{References}

[1] P. Victor, C. Cornelis and M. Cock, "Trust Networks for Recommender Systems", vol. 4, (2011).

[2] J. Bobadilla, A. Hernando and O. Fernando, "Knowledge-Based Systems", vol. 46, (2013).

[3] G. Zaobin, D. Qian, L. Kai and X. Guoqiang, "Journal of Software", vol. 10, no. 22, (2011).

[4] N. Sánchez-Pi, J. Carbó and J.M. Molina, "Knowledge-Based Systems”, vol. 27, (2012).

[5] C. Xi, Z. Zibin, L. Xudong, H. Zicheng and S. Hailong, "IEEE Transactions on services computing", vol. 1, no. 6, (2013).

[6] Z. Qiang and S. Yujiang, Tsinghua University (Natural Science), vol. 3, no. 54, (2014).

[7] W. Haiyan, Y. Wenbin, W. Suichang and L. Sirui, "Journal of Computers", vol. 2, no. 37, (2014).

[8] Z. Peiyun, H. Bo, X. Rongjian and C. Chuanming, "Small micro-computer system", vol. 2, no. 35, (2014).

[9] D. Shuiguang, H. Longtao, W. Jian and W. Chaohui, "Journal Of Computer Science And Technology", vol. 1 , no. 29, (2014). 
[10] Z. Fuguo, "Small micro-computer system", vol. 7, no. 35, (2014).

[11] Z. Yuxiao and Lv Linyuan, "University of Electronic Technology", vol. 2, no. 41, (2012).

[12] J. Golbeck, "Computing And Applying Trust In Web-Based Social Networks", PhDthesis, University of Maryland, USA, (2005).

[13] Hurley N. and Zhang M., ACM Transactions on Internet Technology, vol. 4, no. 10, (2011).

\section{Authors}

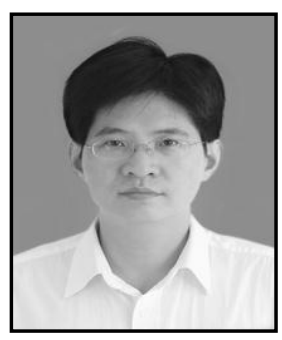

Du Ruizhong, (1975- ), Male, Hebei province, Hebei University professor. information security and trusted computing.

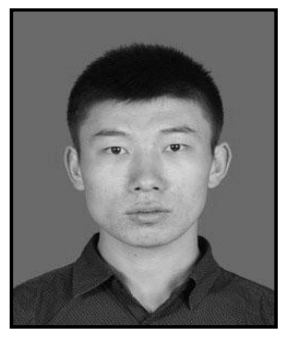

Pei Zhi, (1990- ), Male, HeBei province, Hebei University information security.

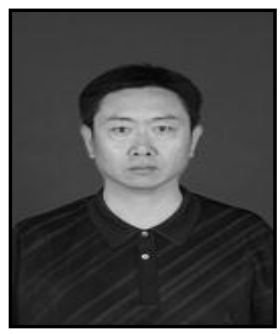

Tian Junfeng, (1965- ), Male, Hebei province, Hebei University professor. network security. 
International Journal of Security and Its Applications

Vol. 10, No. 9 (2016) 